\title{
Review Article \\ Electromagnetic Heating of Heavy Oil and Bitumen: A Review of Experimental Studies and Field Applications
}

\author{
Albina Mukhametshina ${ }^{1,2}$ and Elena Martynova ${ }^{1,2}$ \\ ${ }^{1}$ Harold Vance Department of Petroleum Engineering, Texas A\&M University, 3116 TAMU-407 Richardson Building, \\ College Station, TX, USA \\ ${ }^{2}$ Gubkin Russian State University of Oil and Gas, 65 Leninsky Prospekt, Moscow, Russia
}

Correspondence should be addressed to Elena Martynova; elena.martynova@pe.tamu.edu

Received 11 December 2012; Revised 6 March 2013; Accepted 11 March 2013

Academic Editor: Jorge Ancheyta

Copyright (C) 2013 A. Mukhametshina and E. Martynova. This is an open access article distributed under the Creative Commons Attribution License, which permits unrestricted use, distribution, and reproduction in any medium, provided the original work is properly cited.

\begin{abstract}
Viscosity is a major obstacle in the recovery of low API gravity oil resources from heavy oil and bitumen reservoirs. While thermal recovery is usually considered the most effective method for lowering viscosity, for some reservoirs introducing heat with commonly implemented thermal methods is not recommended. For these types of reservoirs, electromagnetic heating is the recommended solution. Electromagnetic heating targets part of the reservoir instead of heating the bulk of the reservoir, which means that the targeted area can be heated up more effectively and with lower heat losses than with other thermal methods. Electromagnetic heating is still relatively new and is not widely used as an alternate or addition to traditional thermal recovery methods. However, studies are being conducted and new technologies proposed that could help increase its use. Therefore, the objective of this study is to investigate the recovery of heavy oil and bitumen reservoirs by electromagnetic heating through the review of existing laboratory studies and field trials.
\end{abstract}

\section{Introduction}

High-frequency electromagnetic radiation is a relatively new technique for use in enhanced oil recovery methods. It has been tested by theoretic, laboratories and field trial research in Russia [1-10], the United States [11-17], Canada [18-21], and other countries [22-34]. Traditional thermal recovery and well stimulation techniques using hot steam or fluid are not effective in some cases $[7,35]$ due to prohibitive heat losses from injection wells and reservoirs, low reservoir injectivity (especially for bitumen deposits), steam leakage, large overburden heat loss at thin pay zones, permafrost conditions, and so forth. Furthermore, commonly used thermal recovery methods are not considered environmentally friendly, damaging the hydrogeologic environment and contributing to the greenhouse effect.

The most important thing in electromagnetic heating is that the heat is developed within the material rather than being brought from outside, which means the material is heated more uniformly throughout the medium [27]. Therefore, instead of heating the bulk reservoir volume, part of the reservoir can be targeted and heated more effectively with lower heat loss than other thermal methods. Unlike traditional thermal recovery methods, microwave heating causes friction by vibration of molecules, which results in dielectric heating of the reservoir. Heat and mass transfer in different environments under microwave influence was studied by a number of scientists around the globe, but its application as an EOR method is not yet fully understood. Microwave heating is not used productively because of the lack of reliable information about the processes of heat and mass transfer in a multiphase system in porous media under the influence of electromagnetic radiation, which does not allow effective control. Therefore, current research studies use modeling to discover optimum design parameters for the use of microwave heating in field applications. 


\section{Review of Experimental Electromagnetic Heat Studies}

The success of near wellbore heating with electromagnetic waves has been proven experimentally [1]. To represent reservoir rock, quartz sand with a 7.7 dielectric constant and a 0.083 tangent was used in the laboratory. A $20 \%$ initial water saturation and an $80 \%$ initial oil saturation with $16.61 \mathrm{cps}$ $\left(16.61 * 10^{-3} \mathrm{~Pa} \cdot \mathrm{s}\right)$ gravity and $0.86 \mathrm{~g} / \mathrm{cm}^{3}\left(860 \mathrm{~kg} / \mathrm{m}^{3}\right)$ density oil were maintained. The dielectric constant and the loss tangent of the oil sample were 2.23 and 0.019 , respectively. In this study of electric and high-frequency electromagnetic heating of a reservoir model, the temperature of the medium was measured by thermometers located at different points of the experimental setup. In another case, a linear radiator with a length of $87 \mathrm{~cm}$ and a diameter of $19 \mathrm{~mm}$ was placed in the center of the setup. The linear radiator was connected via acoaxial cable to the generator supplying electromagnetic waves with a frequency of $13.56 \mathrm{MHz}$. In the experiment, it was discovered that when fluid temperature was exposed to a high-frequency electromagnetic field at the same distance from the radiation source it was greater than one initiated by electric heating. In this case, the thermal conductivity of the medium is affected only slightly. When heat is induced throughout the whole volume of the medium, the amount of heat introduced depends largely on the electrical properties of the medium. The study summarized the advantages of microwave heating over electrical heating as having deeper penetration, quicker heating, and lower heat losses.

Sayakhov [2] discussed the physical foundations of fluid filtration in high-frequency fields. Radial fluid filtration through porous media in a heterogeneous high-frequency electromagnetic field was studied experimentally. A specially designed core holder, filled with a representative porous medium, was located in a coaxial resonator. A high-frequency wave generator was used to generate mw energy, which was directed to the resonator through cables. Studied liquid came into the porous media through the hose first, then through the lumen of the inner conductor of the resonator dripped into a graduated cylinder. Experiments were started with a 200-300 watts power microwave oven at $2400 \mathrm{MHz}$ frequency and 500 watts vibrational power. Temperatures were recorded throughout the experiments via thermocouples inserted in the core holder. Kerosene was used as a representative reservoir fluid. The measurements were performed with and without the influence of a high-frequency electromagnetic field. It was established that exposure to highfrequency electromagnetic fields leads to a sharp increase in flow rate per unit of time and fluid temperature at the outlet. In addition, the flow rate increases dramatically once exposed to the electromagnetic field, while the temperature increases after 10 seconds. After the field is discontinued, a sharp decrease in the flow rate is observed and a gradual cooling of the porous medium takes place.

Experimental studies [3] on the influence of electromagnetic fields with a frequency of $3 * 10^{5} \mathrm{~Hz}$ to $6 * 10^{5} \mathrm{~Hz}$ on thermal conductivity of dielectric liquids showed that the thermal conductivity of the liquids increased when exposed. The thermal conductivity increases as the magnitude of the dipole moment of the liquid used in the experiment parameters increases with the frequency and intensity of field.

Fatikhov [5] conducted experimental research on the flow of bitumen oil at different pressure gradients in a highfrequency electromagnetic field. The experiment focused on changes in the volumetric flow rate of filtered oil under different pressures at different temperatures. The initial pressure drop for bitumen from the Mordovo-Karmalskoye deposit in reservoir conditions was $0.003 \mathrm{MPa} / \mathrm{m}$. During the experiment, the pressure drop decreased rapidly as the temperatures were increased, resulting in bituminous oil becoming a Newtonian liquid. Therefore, it was established that the application of electromagnetic heating improves fluid flow behavior and the non-Newtonian properties of bitumen decrease rapidly with increasing temperature.

In 1992, Kasevich et al. [17] studied electromagnetic heating of rock samples at $1 \mathrm{~kW}$ (frequency $50.55 \mathrm{MHz}$ ) and $200 \mathrm{~W}(144 \mathrm{MHz})$ and found that a particular type of rock could be heated to $423 \mathrm{~K}$ when exposed to an $\mathrm{RF}$ electromagnetic field. The rock, which had low thermal conductivity, heats poorly when hot steam is pumped into the reservoir. They conducted experiments at both normal and formation pressures.

Ovalles et al. [30] used a microwave with 650 watts power to heat core samples saturated with oil of 25 API gravity and 7.7 API gravity (a sample from the Orinoco River Basin). Medium API oil temperatures were measured in $0.5,1$, and 1.5 minute intervals and heavy oil intervals were increased to 1,5 , and 10 minutes. The experimental results were used to test mathematical models and predict the production of three abstract oil reservoirs in Venezuela.

Chakma and Jha [23] conducted laboratory experiments using electromagnetic heating on a scaled thin heavy oil reservoir pay zone model. Gas injection with horizontal wells during electromagnetic heating was achieved. The aim was to decrease oil viscosity with electromagnetic heating and obtain a gas drive with the injected gas. Using nitrogen for the injected gas, they were able to prove that for thin pay zones heating of the wellbore vicinity is sufficient, by achieving oil recoveries as high as $45 \%$ of original oil in place compared to estimated primary recovery rates of less than $5 \%$. Recovery achieved by use of the combined method was higher than that of nitrogen injection or electromagnetic heating alone. Chakma and Jha also discussed a number of parameters affecting the results of the combined method, including

(i) gas injection pressure (when no gas was injected, oil was produced only due to gravity drainage and no significant convective transport occurred; therefore, gas injection provided an oil rate increase with the increasing injection pressure);

(ii) temperature (the initial production rate was not significantly affected by temperature, but later there was an increase in the production rate, meaning that overall recovery increased with temperature);

(iii) electromagnetic frequency (the higher the frequency, the greater the recovery);

(iv) oil viscosity (as expected, a higher oil viscosity leads to a lower recovery for a given electromagnetic frequency, temperature, and gas injection pressure); 
(v) salinity (higher salinity provides higher recovery due to the higher conductivity of saline water compared with distilled water);

(vi) electrode distance (recovery is similar, but closer electrode spacing provides faster production rates) [23].

Hascakir et al. [24] conducted a laboratory study of microwave-assisted gravity drainage on heavy oil samples from reservoirs in Turkey (Bati Raman, 9.5 API; Garzan, 12 API; and Camurlu, 18 API) using a specially designed novel graphite core holder packed with crushed limestone. Their study described the effects of operational parameters like heating time, waiting period and rock, and fluid properties on the effectiveness of microwave heating. Some of the conclusions made are confirming ones found in Chakma and Jha [23], like the positive effect of high water salinity and water saturation. Hascakir et al. [24] also concluded that water wet conditions are preferable for obtaining higher oil recoveries and that large porosity and permeability are also favorable. When microwave heating is applied to oil samples continuously, higher temperatures are reached, which allows better results to be achieved than with periodic heating when microwave heating is applied for a limited time in periodic intervals. Therefore, higher temperatures allow for better results in continuous heating.

Jha et al. [27] proposed using microwave-assisted gravity drainage (MWAGD) in the Mehsana oil field in India. They heated specially prepared samples with the required characteristics from that field in the laboratory using a microwave with variable power up to 1000 watts operating at $3 \mathrm{GHz}$ frequency, which allowed them to obtain temperature and viscosity profiles of the gravity-drained oil. They described effects of initial oil and water saturations, wettability, porosity, and permeability similar to those found by Hascakir et al. [24] and Chakma and Jha [23].

Jha et al. [27] suggested using MWAGD commercially by drilling one horizontal well and multiple vertical ones with downhole microwave antennas. However, this might not allow deep enough heat penetration, so other options are also proposed such as a combination of two horizontal wells and installing antenna inside the horizontal production well. Vertical separation of the horizontal well pair is approximately 15 meters, which is far more efficient than SAGD in which the separation is around 5 meters. Because crude oil absorbs microwave heat weakly, Jha et al. also proposed increasing thermal conductivity by injecting powdered metallic oxides, chlorides, or activated carbon through a fracture operation. The working principle and description of laboratory applications of such additives to heavy oil can be found in various studies by Hascakir et al., Kershaw et al., and Odenbach $[25,28,29]$.

Technical principles of the SAGD method assisted by electromagnetic heating (EM-SAGD process) were reported by Koolman et al. [26]. Inductive heating was initiated in the laboratory using an EM source with a working frequency of $142 \mathrm{kHz}$. The sample was heated for 10 minutes at a power of $7.2 \mathrm{~kW}$, achieving a rise in the temperature of $7.5 \mathrm{~K}$. Laboratory

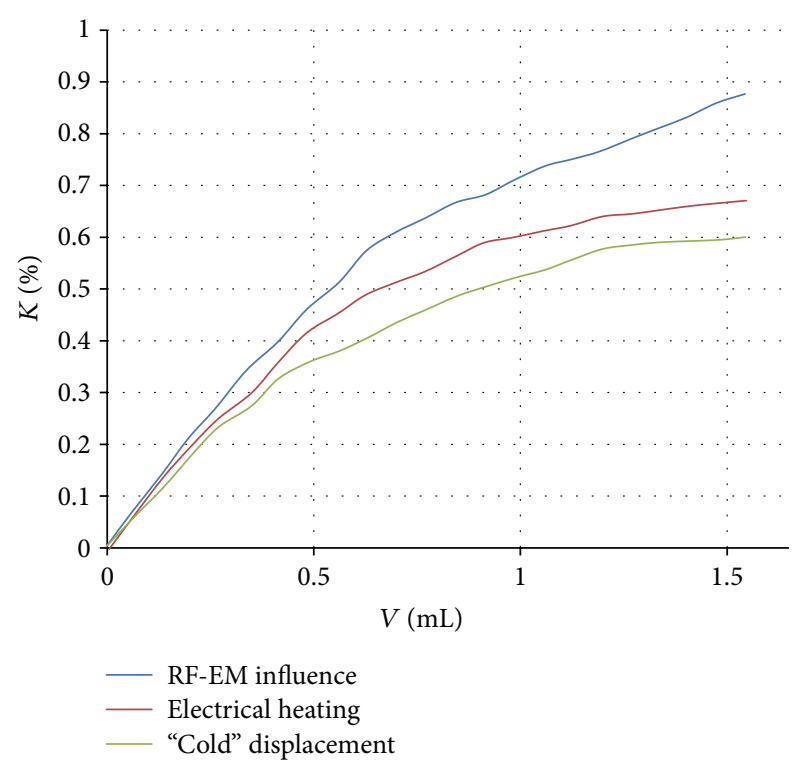

FIGURE 1: Dependence of oil recovery $(K)$ on the relative volume of solvent injected. Adapted from-[6].

and field processes were modeled using a numerical simulator, combining electromagnetic and thermal modules. It was specially built and can be applied to field-scale simulations. According to simulation results, a 38\% increase in bitumen production was predicted compared to conventional SAGD.

Kovaleva et al. investigated the effects of radio frequency electromagnetic (RF-EM) fields and electrical heating on the mass- and heat-transfer processes in a multicomponent hydrocarbon system flowing in porous media $[4,6]$. Three different types of experiments were carried out: solvent (kerosene) flooding under the RF-EM field, solvent (kerosene) flooding under electrical heating, and cold solvent (kerosene) flooding. In all three experiments, the physical characteristics of the model and heating conditions (temperatures) were identically maintained. Two series of experiments on models with different granulometric composition of formation were also carried out.

Figure 1 shows the dependence of oil recovery on the volume of solvent injected. This figure demonstrates that the highest oil recovery was obtained by applying an RF-EM field. Kovaleva et al. $[4,6]$ concluded that following RF-EM influence on the oil-saturated samples the quantity of the received oil is more than the quantity received under electrical (and thermal) processing at identical temperatures of heating of the media. It confirms the additional "nonthermal" action of the electromagnetic field.

\section{Technologies of In Situ Electromagnetic Heating of Heavy Oil and Bitumen}

The first production method applying microwave heating to well production was patented in 1956 [14]. Electromagnetic waves were transferred to the well bottom from the surface through a coaxial system of internal and external pipes (tubing and casing). Interaction of electromagnetic waves with 
TABLE 1: Characteristics of Well 40/19 of the Ishimbayskoye Oil Field.

\begin{tabular}{lc}
\hline Parameter & Value \\
\hline Depth, $\mathrm{m}$ & 830 \\
Casing diameter, in. & 6 \\
Tubing diameter, in. & 2 \\
Flow rate, ton/day & 3 \\
Well temperature, $\mathrm{K}$ & $287-289$ \\
Paraffin content, $\%$ & 2.3 \\
Resins content, $\%$ & 11 \\
Density, $\mathrm{kg} / \mathrm{m}^{3}$ & 890 \\
Viscosity at $20^{\circ} \mathrm{C}, \mathrm{m}^{2} / \mathrm{c}$ & $20 * 10^{-6}$ \\
\hline
\end{tabular}

Sayakhov et al. [10].

the formation causes the emergence of distributed volumetric heat sources and reduces the viscosity of the reservoir fluid. In 1965, Haagensen [13] described a device for generating high-frequency electromagnetic waves at the mouth of the well and a method of delivering electromagnetic energy through coaxial lines and waveguides to the bottom hole. In 1987, Wilson [16] described a similar device in his work, with some modifications of the radiating element of EM waves.

A huge drawback to the methods described by Haagensen and Wilson $[13,16]$ is the shallow penetration of electromagnetic waves, and, hence, a low sweep efficiency of heating. When the method described by Ritchey [14] was implemented, there were large losses of electromagnetic energy. Due to the finite conductivity of tubing, they are heated and electromagnetic energy is dissipated in rocks surrounding the well, resulting in large wellbore heat losses, especially if a permafrost layer is present.

Sayakhov et al. [8] proposed a method of recovery that included creating a combustion front with a simultaneous electromagnetic current influence. The environment is heated when exposed to an electromagnetic field, which decreases the viscosity and increases the mobility of crude oil. It is assumed in this method that the EM field continues influencing the reservoir after the combustion is initiated.

3.1. Review of Electromagnetic Heating Field Studies. Electromagnetic heating field trials have been carried out in Russia (Bashkortostan and Tatarstan) [2, 9, 10], the United States (California and Utah) [11, 17], and in Canada (Alberta and Saskatchewan) [18-21].

3.1.1. Russia. In Russia, field tests of radio frequency electromagnetic heating of the near-wellbore zone were first launched in 1969 at Well 40/19 in the Ishimbayskoye Oil Field in Bashkortostan and continued in the Yultimirovskoye Bitumen Field in Tatarstan according to Sayakhov et al. [2, 9, 10]. Characteristics of Well 40/19 from the Ishimbayskoye Oil Field are represented in Table 1.

The source of high-frequency electromagnetic energy was a generator providing an optimum oscillation output power of $63 \mathrm{~kW}$ at a frequency of $13.56 \mathrm{MHz}$. Standard RF coaxial cable was used to supply high-frequency electromagnetic
TABLE 2: Dynamics of temperature growth.

\begin{tabular}{lc}
\hline Heating time, days & Temperature increase at the bottom hole, K \\
\hline 0.5 & 283 \\
1 & 290 \\
2 & 300 \\
3 & 306 \\
4 & 311 \\
5 & 313 \\
\hline
\end{tabular}

Sayakhov et al. [9].

TABLE 3: Characteristics of the Yultimirovskoye reservoir.

\begin{tabular}{lc}
\hline Parameter & Value \\
\hline Porosity, \% & 25 \\
Bitumen saturation, \% & 3.6 \\
Permeability, micm $^{2}$ & $0-0.183$ \\
\hline
\end{tabular}

Sayakhov [2].

energy from the generator to the well. Temperature measurements were carried out using a thermograph at the bottom hole. Temperature was recorded continuously at a fixed depth of 650-655 m (in the open hole, where the radiating element was working) while the generator was operating (Table 2).

3.1.2. Yultimirovskoye Bitumen Field. RF electromagnetic heating was conducted in the Yultimirovskoye Bitumen Field by Sayakhov in 1980 [2] (Table 3). Two wells spaced 5 meters apart were studied, Well 150 and Well 1.

Electromagnetic heating of the bitumen reservoir was conducted in several stages at different conditions. Initially, the RF-EM installation was set to about of $20 \mathrm{~kW}$ power. After 36.5 hours, the temperature at the bottom of Well 150 has increased from 282 to $389 \mathrm{~K}$. No temperature change was observed in Well 1. In the next phase, RF-EM installation was reset to approximately $30 \mathrm{~kW}$ of power. As a result, the temperature in Well 150 reached $423 \mathrm{~K}$ after six hours. It should be noted that the growth rate of temperature increased. In the third phase, the RF-EM installation was set to a maximum of $60 \mathrm{~kW}$, which caused the heating intensity in Well 150 to increase greatly. After 5.5 hours, the temperature in Well 150 increased from 417 to $463 \mathrm{~K}$. Next, the RF-EM unit was turned off for 2 hours, resulting in a temperature drop to $423 \mathrm{~K}$.

During the next 32 hours, the RF-EM installation operated at maximum output, causing the heating of the bottom hole of Well 150 up to $583 \mathrm{~K}$ and the bottom hole of Well 1 up to 318 K. In Sayakhov's experiment [2], the fluoroplastic collars that centered the tubing in Well 150 melted (their maximum operating temperature was $573 \mathrm{~K}$ ). As a result, a short circuit between the casing and tubing occurred and the RF-EM unit broke and was disabled. Before this disruption, the RF-EM installation worked steadily throughout the field experiment.

Deep heat penetration (up to $5 \mathrm{~m}$ in the reservoir) was demonstrated by temperature measurements done during the cooling of Well 150's drain zone after the exposure. The temperature decreased from $373 \mathrm{~K}$ to $343 \mathrm{~K}$ in three days. 
After a few days of electromagnetic heating, wellbore heat distribution revealed low heat losses to both the overburden and underburden.

\subsection{The United States}

3.2.1. Bakersfield, California, United States. In 1992, Kasevich et al. [17] conducted field tests of RF-EM heating in the United States in the Bakersfield, California, field. The goal was to prove the concept that controlled RF-EM radiation could be used as a thermal EOR method. The production of reservoir fluids was not measured because it was a quality, not quantity, study designed to gain a better understanding of underground processes.

A high-frequency electromagnetic wave generator with a capacity of $25 \mathrm{~kW}$ and frequency of $13.56 \mathrm{MHz}$ was used to heat the reservoir at Well 100D. Heat penetration was determined by temperature measurements in the surrounding observation wells T10, T20, and T30, which were located 3, 6, and 9 meters from Well 100D. Kasevich et al. [17] also proved that the RF producer used could efficiently focus its radiation pattern into the desired region by measuring return loss and electromagnetic radiation. In well T10, situated 3 meters from Well 100D, the medium temperature was increased from $293 \mathrm{~K}$ to $393 \mathrm{~K}$ over 20 hours of EM heating.

3.2.2. Avintaquin Canyon and Asphalt Ridge (Utah). In 1980, one of the most detailed studies on electromagnetic-heatbased oil recovery was done at the Illinois Institute of Technology Research Institute (IITRI) by Bridges et al. [11]. They carried out extensive research work on the use of the different types of electromagnetic heating for different types of deposits, oil shale, and tar sand.

Bridges et al. [11] tested their IITRI technique of RF electromagnetic heating with two field experiments in Avintaquin Canyon, Utah, USA. Shale that was 6 meters thick was found in outcrops convenient for relatively cheap horizontal experiments. So arrays of holes were drilled and electrodes were inserted to a depth of 1 meter. These tests allowed the researchers to gain experience and to prove it was possible to achieve in situ pyrolysis of oil shale, thus increasing its thermal maturity. The power applied to the formation ranged from $5 \mathrm{~kW}$ to $20 \mathrm{~kW}$, with a frequency of $13.56 \mathrm{MHz}$. As a result of EM heating, temperatures rose to $673 \mathrm{~K}$ and $20-$ $30 \%$ of the oil content was collected. However, it should be noted that the amount of produced oil was badly affected by the presence of cracks which allowed light hydrocarbons to escape (evaporate).

In 1981, Bridges et al. [11] conducted field tests on tar sand at Asphalt Ridge, Utah, USA. The first experiment tested the gravity drive bitumen recovery process, designed to prove EM heating concepts and improve equipment design. This experiment used vertical electrode placement and a mined collection chamber and tunnel. It was equipped with a $200 \mathrm{~kW}$ radio transmitter and heated $25 \mathrm{~m}^{3}$ of tar sand. In the first experiment, the roof of the mined chamber was not supported well enough, which resulted in early termination of the experiment. Therefore, the situation had to be fixed by constructing a concrete arch for the second pilot test.
TABLE 4: Characteristics of the reservoir in the Wildmere field, Alberta, Canada.

\begin{tabular}{lc}
\hline Parameter & Value \\
\hline Net thickness, $\mathrm{m}$ & 1 \\
Depth, $\mathrm{m}$ & 600 \\
Density, $\mathrm{kg} / \mathrm{m}^{3}$ & 987 \\
Oil viscosity at $20^{\circ} \mathrm{C}, \mathrm{Pa} \cdot \mathrm{s}$ & 20 \\
\hline
\end{tabular}

Spencer [18].

The heating power used on tar sand varied from $40 \mathrm{~kW}$ to $75 \mathrm{~kW}$ with a frequency of $13.56 \mathrm{MHz}$.

The second test quantified the results of heating over a longer period and at higher temperatures. In this experiment, temperatures exceeded $473 \mathrm{~K}$, and 30 to $35 \%$ recovery was achieved in just 20 days. This was encouraging because continuation of heating could have resulted in even higher recovery. The power loss was minimal in all the experiments, which proved the efficiency of heating.

\section{Canada}

4.1. The Wildmere Field, Alberta, Canada. According to Spencer [18] commercial EM heating was first introduced in the field at Wildmere, Alberta, Canada (Table 4). The first well was drilled in January 1986 and began producing oil in March of the same year. Before EM heating began in May, the well was producing about 0.95 tonnes/day. After EM heating commenced, production rates increased and soon settled at the level of 3.18 tonnes/day until November 1986, when the well was closed due to technical reasons. Another well in this field increased in production from 1.59 tonnes/day to an average of 4.77 tonnes/day, with the maximum flow rate reaching 9.54 tonnes/day.

4.2. The Lloydminster Heavy Oil Area, Saskatchewan, Canada. In 1988-1989, two electromagnetic stimulation projects were conducted by Davidson [22] in the Lloydminster heavy oil area in Saskatchewan, Canada. Unfortunately the economic potential of the process could not be evaluated from either pilot test, because long-term heating could not be achieved due to equipment failure (casing insulation) and special reservoir conditions. However, the technical results looked promising.

The first pilot well was in Northminster (Saskatchewan, Canada) and produced 11.4 API oil from the Sparky formation. The power was applied to the well in a pulsating manner with a baseline of $20 \mathrm{~kW}$ with four-hour spikes of $30 \mathrm{~kW} \mathrm{(2}$ daily), in order to reduce the risk of significant damage to the insulation. Later, the power was increased to a $25 \mathrm{~kW}$ baseline with $35 \mathrm{~kW}$ pulses and finally to a $30 \mathrm{~kW}$ baseline with $50 \mathrm{~kW}$ peaks. At that point the insulation failed, and the power rates came down to $28 \mathrm{~kW}$. Power rates were later leveled at $47 \mathrm{~kW}$ and stayed that way until terminated.

As can be seen from Table 5, water cut and production both reacted positively to electromagnetic stimulation. However, it should be noted that some portion of the increased 
TABLE 5: Oil field performance for the Northminster pilot: primary and achieved by EM heating.

\begin{tabular}{lcc}
\hline Parameter & Primary & EM heating \\
\hline Production rate, $\mathrm{m}^{3} /$ day & $10-12$ & 20 \\
Water cut, \% & $15-20$ & $10-12$ \\
Productivity index, bbl/psi & 0.33 & 0.42 \\
Stimulation ratio & & 1.27 \\
\hline
\end{tabular}

Davidson [22].

production is related to the increase in pump speed. Water cut drop can be related directly to the EM effect and improvement in oil mobility. Once the heating has been terminated, technical parameters return rapidly to their initial states.

The second pilot well was situated in Lashburn (Saskatchewan, Canada) and produced very viscous 11.4 API crude oil from the Sparky formation. During the reservoir heating phase, the power ranged from 13 to $18 \mathrm{~kW}$. This well has high sand cuts and before electrical power was applied its production was not stable and regularly had to be stimulated by flushing the wellbore. The peak production reached $5.0 \mathrm{~m}^{3} /$ day and had begun to decline before electromagnetic heating was applied. Electromagnetic heating reduced the water cut, but the well was still prone to high water cuts after shut-in periods. Oil production also increased when electromagnetic forces were applied, until it reached $9 \mathrm{~m}^{3} /$ day. During the initial heating phase of the reservoir, the temperature in the bottom hole increased steadily from $295 \mathrm{~K}$ to $309 \mathrm{~K}$, but temperatures began dropping immediately after the power was turned off or when power delivery systems failed.

4.3. ESEIEH (Alberta, Canada). Enhanced Solvent Extraction Incorporating Electromagnetic Heating technology (ESEIEH) has been patented and is currently undergoing tests, as reported by Rassenfoss [20]. The ESEIEH consortium is relying on three oil company partners to help with this testing: Laricina Energy, Nexen, and Suncor Energy. The pilot project is planned to take three years and currently is in the first stage. Field application is expected to start later in 2013. The ESEIEH method combines the familiar horizontal well pair design commonly used in the Canadian oil sands, coupled with heating using RF-EM waves and solvents, such as butane or propane. The company aims to heat the reservoir by running an antenna underground that emits enough energy to raise the temperature to $50^{\circ} \mathrm{C}\left(120^{\circ} \mathrm{F}\right)$.

\section{Conclusion}

A review of electromagnetic heating for enhanced oil recovery was presented in this paper. A number of studies show that electromagnetic heating is a promising method of enhanced oil recovery. However, the studies to date are limited, and only a few field trials have been reported. Most of the current research is based on laboratory experiments or numerical models. It should be noted that this paper did not cover the computer simulations carried out to research the effectiveness of EM heating.

Better understanding of the in situ electromagnetic process is essential and can be achieved by combining laboratory, numerical, and field-scale tests. At the moment it is not possible to assess the efficiency of EM heating or the opportunities for economic applications of it alone or in combination with traditional methods; therefore, more global studies should be conducted.

Even though sustainability of this technology has not yet been completely evaluated, the method definitely should not be overlooked by the industry because of its enormous potential. Attempts should be made to develop viable screening criteria for possible production of heavy oil, oil shale, and tar sand deposits.

\section{References}

[1] S. Chistyakov, F. Sayakhov, and G. Balabyan, "Experimental study of formations dielectric properties under the influence of high-frequency electromagnetic fields," in University Investigations: Geology and Exploration, pp. 153-156, 1971.

[2] F. Sayakhov, "Particular properties of filtration and fluid flow under the influence of high-frequency electromagnetic field," in Joint University Scientific Book, pp. 108-120, 1980.

[3] B. Savinikh, V. Dyakonov, and A. Usmanov, "The influence of alternating electric currents on the thermal conductivity of dielectric fluids," Journal of Engineering Physics and Thermophysics, no. 2, pp. 269-276, 1981 (Russian).

[4] A. Davletbaev and L. Kovaleva, "Combined RF EM/solvent treatment technique: heavy/extra-heavy oil production model case study," in Proceedings of the 10th Annual International Conference Petroleum Phase Behavior and Fouling, Rio de Janeiro, Brazil, 2009.

[5] M. A. Fatikhov, "Experimental study of bitumen initial pressure gradient in the electromagnetic field," University Investigations: Oil and Gas, no. 5, pp. 93-94, 1990 (Russian).

[6] L. Kovaleva, A. Davletbaev, T. Babadagli, and Z. Stepanova, "Effects of electrical and radio-frequency electromagnetic heating on the mass-transfer process during miscible injection for heavy-oil recovery," Energy and Fuels, vol. 25, no. 2, pp. 482486, 2011.

[7] G. Malofeev, O. Mirsaetov, and I. Cholovskaya, "Injection of hot fluids for enhanced oil recovery and well stimulation," in Regular and Chaotic Dynamics, Institute of Computer Science, RussiaIgevsk, Russia, 2008.

[8] F. Sayakhov, R. Bulgakov, V. Dyblenko, B. Deshura, and M. Bykov, "About HF heating of bitumen reservoirs," Petroleum Engineering, no. 1, pp. 5-8, 1980 (Russian).

[9] F. L. Sayakhov, L. A. Kovaleva, M. A. Fatikhov, and G. A. Khalikov, "Method of thermal effect on oil-bearing formation," SU Patent 1723314, 1992.

[10] F. Sayakhov, I. Habibullin, M. Yagudin, and M. Fatyhov, "Technique and technology of thermal well stimulation on the basis electro-thermo-chemical and electromagnetic effects," University Investigations: Oil and Gas, no. 2, pp. 33-42, 1992 (Russian).

[11] J. E. Bridges, J. J. Krstansky, A. Taflove, and G. C. Sresty, “The IITRI in situ RF fuel recovery process," Journal of Microwave Power, vol. 18, no. 1, pp. 3-14, 1983.

[12] J. Bridges, "Method for in-situ heat processing of hydrocarbonaceous formation," US Patent 4140180, 1979. 
[13] A. D. Haagensen, "Oil well microwave tools," Patent USA 3170119, 1965.

[14] H. W. Ritchey, "Radiation Heating System, US Patent," Tech. Rep. 2757738, 1956.

[15] G. C. Sresty, R. H. Snow, and J. E. Bridges, "Recovery of liquid hydrocarbons from oil shale by electromagnetic heating insitu," US Patent 4485869, 1984.

[16] R. Wilson, "Well production method using microwave heating," US Patent 4485868, 1987.

[17] R. S. Kasevich, S. L. Price, D. L. Faust, and M. F. Fontaine, "Pilot testing of a radio frequency heating system for enhanced oil recovery from diatomaceous earth," in Proceedings of the SPE Annual Technical Conference \& Exhibition, pp. 105-113, New Orleans, La, USA, September 1994.

[18] H. L. Spencer, "Electromagnetic Oil Recovery, Ltd," Calgary, Canada, 1987.

[19] F. E. Vermeulen and F. S. Chute, "Electromagnetic techniques in the in-situ recovery of heavy oils," Journal of Microwave Power, vol. 18, no. 1, pp. 15-29, 1983.

[20] S. Rassenfoss, "Seeking more oil, fewer emissions," Journal of Petroleum Technology, vol. 64, no. 9, pp. 34-38, 2012.

[21] B. C. W. Mcgee and F. E. Vermeulen, "The mechanisms of electrical heating for the recovery of bitumen from oil sands," Journal of Canadian Petroleum Technology, vol. 46, no. 1, pp. 2834, 2007.

[22] R. J. Davidson, "Electromagnetic stimulation of Lloydminster heavy oil reservoirs: field test results," Journal of Canadian Petroleum Technology, vol. 34, no. 4, pp. 15-24, 1995.

[23] A. Chakma and K. N. Jha, "Heavy-oil recovery from thin pay zones by electromagnetic heating, paper SPE 24817," in Proceedings of the Annual Technical Conference and Exhibition, Society of Petroleum Engineers, Washington, DC, USA, October 1992.

[24] B. Hascakir, C. Acar, Schlumberger, B. Demiral, and S. Akin, "Microwave assisted gravity drainage of heavy oils," in Proceedings of the International Petroleum Technology Conference (IPTC '08), pp. 1908-1916, Kuala Lumpur, Malaysia, December 2008.

[25] B. Hascakir, T. Babadagli, and S. Akin, "Experimental and numerical modeling of heavy-oil recovery by electrical heating, paper SPE 117669," in Proceedings of the International Thermal Operations and Heavy Oil Symposium (ITOHOS '08), p. 14, Society of Petroleum Engineers, Alberta, Canada, October 2008.

[26] M. Koolman, N. Huber, D. Diehl, and B. Wacker, "Electromagnetic heating method to improve steam assisted gravity drainage, paper 1177481," in Proceedings of the International Thermal Operations and Heavy Oil Symposium (ITOHOS '08), pp. 327-338, Society of Petroleum Engineers, Alberta, Canada, October 2008.

[27] K. A. Jha, N. Joshi, and A. Singh, "Applicability and assessment of micro-wave assisted gravity drainage (MWAGD) applications in Mehsana heavy oil field, paper SPE 14591," in Proceedings of the SPE Heavy Oil Conference and Exhibition, Society of Petroleum Engineers, Kuwait City, Kuwait, December 2011.

[28] J. R. Kershaw, G. Barrass, and D. Gray, "Chemical nature of coal hydrogenation oils part I. The effect of catalysts," Fuel Processing Technology, vol. 3, no. 2, pp. 115-129, 1980.

[29] S. Odenbach, "Ferrofluids-magnetically controlled suspensions," Colloids and Surfaces A, vol. 217, no. 1-3, pp. 171-178, 2003.

[30] C. Ovalles, A. Fonseca, A. Lara et al., "Opportunities of downhole dielectric heating in Venezuela: three case studies involving medium, heavy and extra-heavy crude oil reservoirs, paper SPE
78980," in Proceedings of the International Thermal Operations and Heavy Oil Symposium and International Horizontal Well Technology Conference, Alberta, Canada, November 2002.

[31] M. A. Ayrapetyan, "About oil fields development prospects by high-frequency currents electrical fields," in Materials of KSSR Institute of Oil, pp. 38-52, 1958.

[32] M. A. Ayrapetyan, V. S. Velikanov, and E. Ya. Magnikov, "Reservoir high-frequency heating investigations," in Materials of KSSR Institute of Oil, pp. 113-124, 1959.

[33] M. A. Carrizales, L. W. Lake, and R. T. Johns, "Production improvement of heavy-oil recovery by using electromagnetic heating, paper SPE 115723," in Proceedings of the SPE Annual Technical Conference and Exhibition (ATCE '08), Denver, Colo, USA, September 2008.

[34] A. D. Hiebert, F. E. Vermeulen, F. S. Chute, and C. E. Capjack, "Numerical simulation results for the electrical heating of Athabasca oil-sand formations," SPE Reservoir Engineering, vol. 1, no. 1, pp. 76-84, 1986.

[35] J. Burge, P. Surio, and M. Combarnu, Thermal Methods of Enhanced Oil Recovery, Nedra Publishing, Moscow, Russia, 1988. 

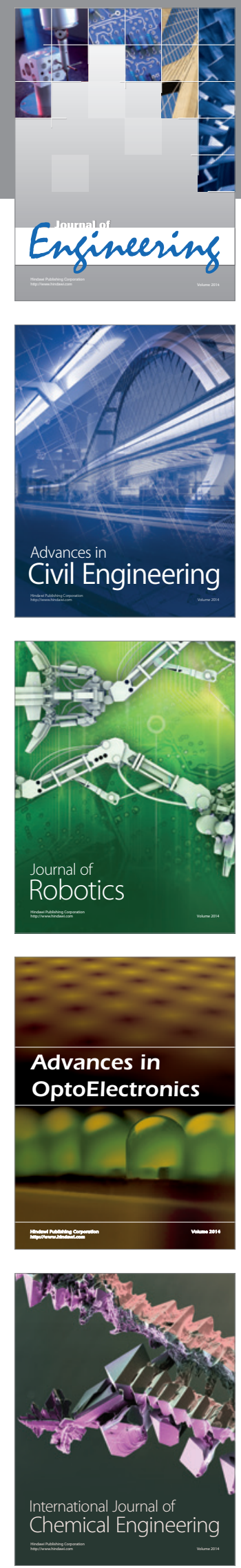

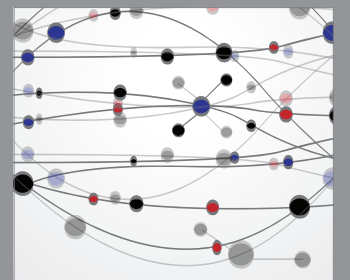

The Scientific World Journal
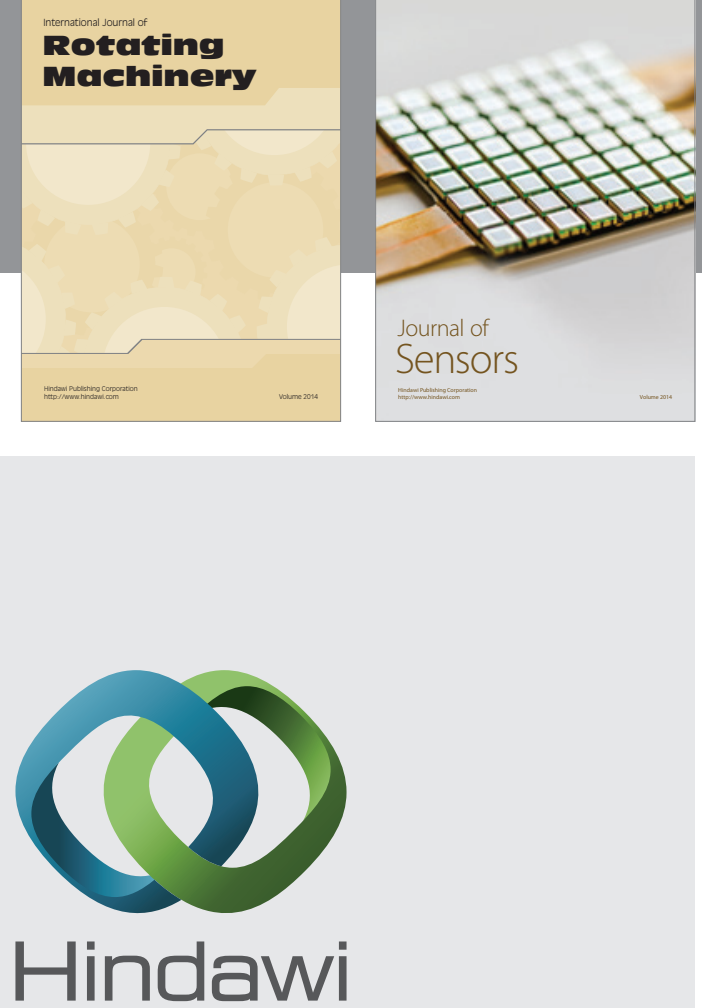

Submit your manuscripts at http://www.hindawi.com
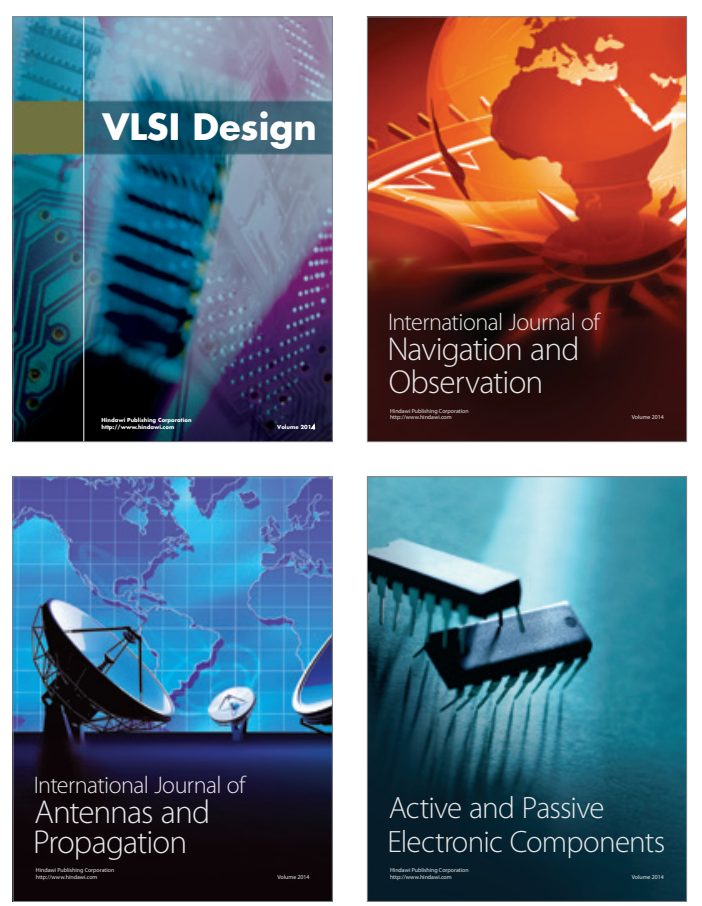
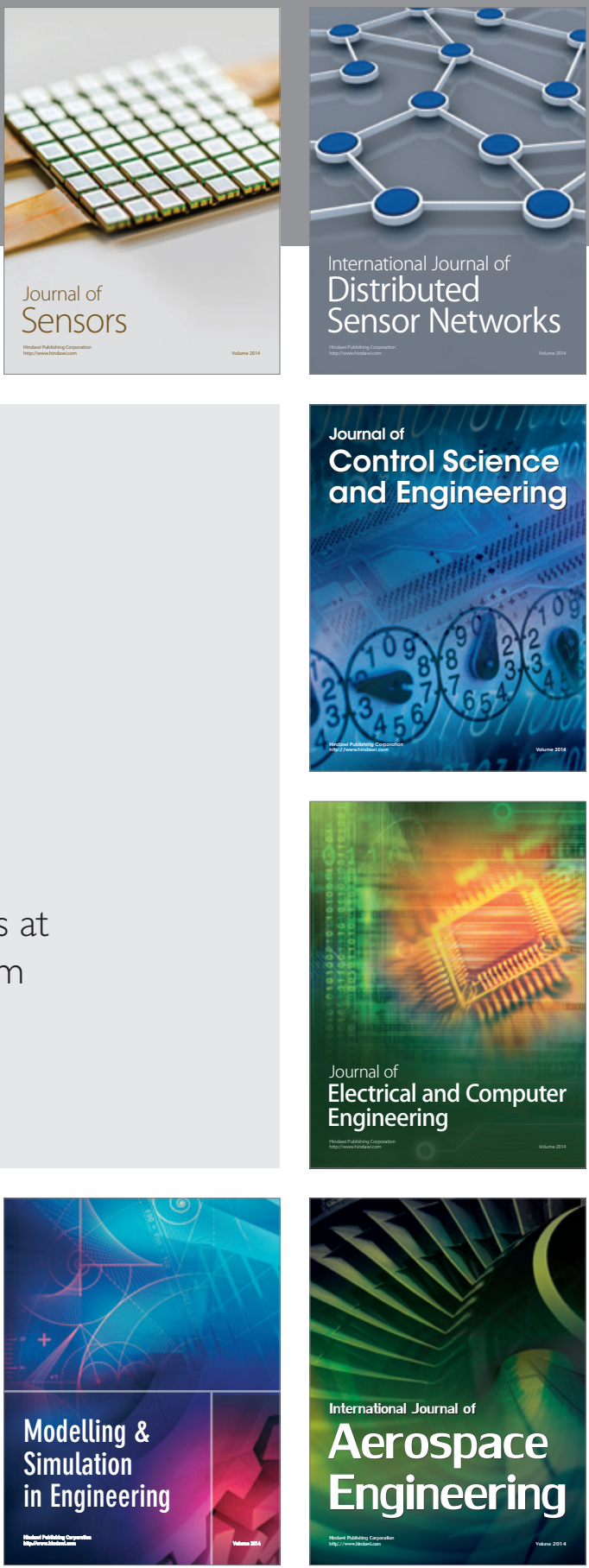

Journal of

Control Science

and Engineering
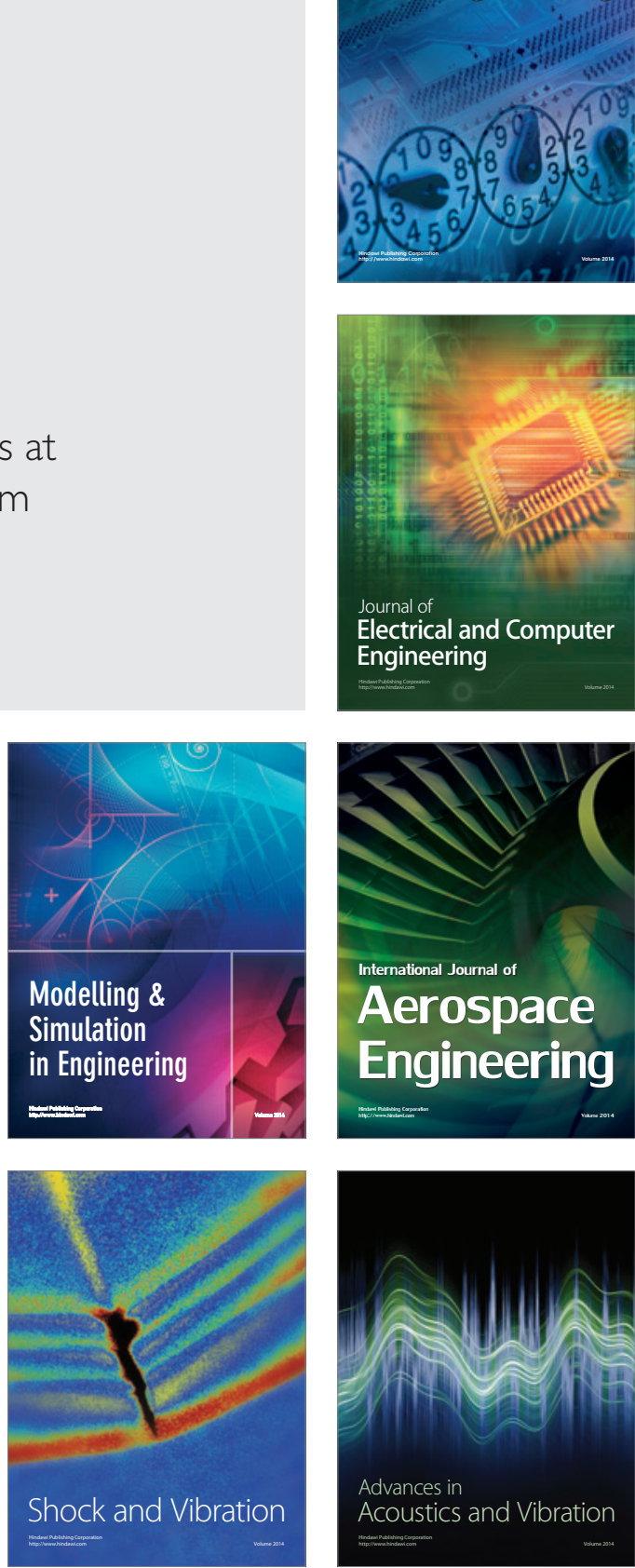